\title{
O uso de parâmetros morfométricos como potencial indicador de ocorrência de fluxos de detritos no litoral norte de São Paulo
}

Tulius Dias Nery*

\section{Resumo}

O objetivo deste trabalho foi avaliar o papel dos parâmetros morfométricos (areal e linear) (métodos qualitativo), associado com as variáveis de precipitação e concentração de sedimentos (método quantitativo), como indicador do potencial de geração de fluxos de detritos em escala de sub-bacias na Serra do Mar, no Litoral Norte do estado de São Paulo. A aplicação de diferentes cenários de precipitação (60 e 100 mm.h-1) e de concentração de sedimentos $(30 \%$ e $50 \%)$ resultou no aumento de $60 \%$ no volume de sedimento em função do acréscimo da intensidade de precipitação. Em relação a concentração de sedimentos, o aumento foi de $71 \%$. Acredita-se que este trabalho possa auxiliar no planejamento dessas sub-bacias e servir como indicador de possíveis ações mitigadoras, minimizando os danos que possam ocorrer próximas ou nas áreas inseridas dentros dessas localidades. Palavras-chaves: Serra do Mar; Ffluxos de detritos; Modelos empíricos.

Analista Operacional em Geociências, Centro Nacional de Monitoramento e Alertas de Desastres Naturais - Cemaden (tulius.nery@cemaden.gov.br).

Geosul, Florianópolis, v. 32, n. 63, p 179-200, jan./abr. 2017 
NERY, T.D. O uso de parâmetros morfométricos como potencial ...

The parameters morphometric potential use as indicator of the debris flows occurrence in Costal North of São Paulo

\begin{abstract}
The objective of this study was to evaluate the role of morphometric (areal and linear) (qualitative method), associated parameters with the rainfall variables and sediment concentration (quantitative method), as a potential indicator of debris flow scale sub-basins in the Serra do Mar, on the north coast of São Paulo. The application of different scenarios of rainfall $(\mathrm{mm} / \mathrm{h})$ and solids concentration (30\% and 50\%) resulted in 60\% increase in sediment volume as a function of intensity of precipitation increase. Concerning sediment concentration, the increase was $71 \%$. It is believed that this work can assist in the planning of that sub-basins and serve as an indicator of possible mitigating actions, minimizing the damage that may occur on or near the areas inserted insides of such sites.
\end{abstract}

Key words: Serra do Mar; Debris flow; Empirical models.

\title{
Introdução
}

Os fluxos de detritos são processos de grande magnitude associados a ambientes montanhosos e são registrados em diversos lugares do mundo. Eles são considerados fenômenos devastadores devido à sua velocidade e ao seu extenso raio de alcance, bem como aos danos e às fatalidades causados por estes processos (DOWLING e SANTI, 2014).

Os fluxos de detritos estão condicionadas a ocorrência de um ou mais escorregamentos e/ou devido à inundação do canal permitindo o deslocamento de sedimentos de diâmetros variados por grandes distâncias (TAKAHASHI, 1981; RICKNMANN e ZIMMERMANN, 1993). Estudos em diferentes partes do mundo verificaram que a concentração de sedimentos ou de sólidos, ou 
NERY, T.D. O uso de parâmetros morfométricos como potencial ...

seja, a fração sedimentar das corridas de detritos, obtida por meio de observações realizadas nos depósitos destes processos, pode variar entre $10 \%$ a $90 \%$ (COSTA, 1984; DAVIES et al., 1992; WHIPPLE e DUNNE, 1992; RICKNMANN e ZIMMERMANN, 1993; JISHAN e TIANCHI, 2001; GODT e COE, 2007 entre outros). Esta variação granulométrica pode interferir no impacto que estes processos podem causar permitindo, por exemplo, danos parciais ou totais nas infraestruturas (GRAMANI et al., 2005).

No entanto, pesquisas a respeito desta dinâmica são restritas, direcionadas, em sua maior parte, a eventos de grande magnitude (Pellerin et al., 1997; Avelar et al., 2006; Kobiyama et al., 2010; Gramani, 2015), e/ou quando atingem e danificam infraestruturas (vias de circulação, estradas férreas, oleodutos, adutoras, etc.) (KANJI et al., 2008; GRAMANI, 2013).

Ainda, o pouco conhecimento da dinâmica destes processos pode ser decorrente da dificuldade de obtenção de dados inviabilizando a construção de um banco de dados, bem como, devido as suas características de deflagração, muitas vezes, ocorrendo em locais de díficil acesso. Consequentemente a estas implicações métodos qualitativos e quantitativos vêm sendo empregados com intuito de compreender estes fenômenos. Tais métodos levam em consideração as variáveis morfométricas da bacia, a intensidade de precipitação, os aspectos litológicos, entre outros (COROMINAS, 1996; RICKENMANN, 1999; MARCHI e D'AGOSTINO, 2004; WILFORD et al. 2004; GARTNER et al., 2008; KANJI et al. 2008), com intuito de estimar o seu raio de alcance, a sua velocidade, a sua vazão de pico e o volume gerado pelos fluxos de detritos. Em outras palavras, conforme Cannon e Gartner (2005), esta relação entre a vazão, as características da bacia de drenagem e a precipitação pode ser útil na previsão do potencial de geração dos fluxos de detritos.

No Brasil, estes processos ocorrem principalmente na faixa leste continental, mais precisamente no compartimento denominado, de forma geral, de Serra do Mar, abrangendo as regiões Sul e Sudeste. Nestas regiões, a ocorrência destes 
NERY, T.D. O uso de parâmetros morfométricos como potencial ...

processos está associada à intensidade e/ou acumulado de precipitação somada a disponibilidade de material, tanto em suas encostas, quanto nos canais de drenagem.

Pode-se destacar, por exemplo, a porção da Serra do Mar localizada no município de Caraguatatuba (SP), a qual vivencia um ciclo destes fenômenos. Mesmo sendo os escorregamentos os processos mais recorrentes na paisagem, são os fluxos de detritos que causam maiores impactos (perdas e danos) quando da sua ocorrência. Exemplo desta dinâmica foi observado no ano de 1967, quando após meses quase ininterruptos de precipitação, ao longo das bacias que compõem o município, foram deflagrados movimentos de massa generalizados.

No país, os fluxos de detritos vêm recebendo maior atenção nos últimos anos por técnicos e pesquisadores de diferentes áreas, após eventos significativos deflagrados em Santa Catarina (principalmente no Vale do Itajaí, em 2008), Rio de Janeiro (região serrana, em 2011), Paraná (Serra do Mar paranaense, 2011) e São Paulo, este último destacando-se os eventos mais recentes ocorridos em Cubatão (2013) e em Itaóca (2014).

Neste contexto, após sucessivos eventos geodinâmicos, tais como, os fluxos de detritos, tendo como ápice os eventos ocorridos na região serrana do Rio de Janeiro, em 2011, foi instituída a Lei $\mathrm{n}^{\circ} 12.608$ de 10 de abril de 2012. A lei vem para estabelecer ações preventivas e mitigadoras à minimização dos desastres associados a movimentos de massa, na qual, uma destas premissas é avaliar a suscetibilidade dos desastres naturais, em escala de bacias hidrográficas.

A partir deste cenário, este trabalho objetiva avaliar a influência de parâmetros morfométricos, associado com as variáveis de precipitação e concentração de sedimentos, como indicador do potencial de geração de fluxos de detritos em escala de sub-bacia na Serra do Mar, especificamente em Caraguatatuba (tendo como base o evento de 1967), no Litoral Norte do estado de São Paulo. Devido à forma de ocupação nestas bacias e sub-bacias, principalmente nas zonas de passagem e de deposição dos fluxos 
NERY, T.D. O uso de parâmetros morfométricos como potencial ...

de detritos, há necessidade de estudos que possam direcionar ações voltadas à minimização de desastres e contribuir no planejamento destas áreas.

\section{Área de estudo}

O município de Caraguatatuba (Figura 1) é sustentando por rochas metamórficas e ígneas, entre elas, granitos, gnaisses, migmatitos e micaxistos, apresentando relevo dissecado, com encostas íngremes (de declividade média superior a $25^{\circ}$ ), com altitudes próximas a $1000 \mathrm{~m}$, alta densidade de drenagem e vales encaixados associados as falhas, fraturas e contatos litológicos (ROSS e MOROZ, 1997). O relevo é compartimentando em três setores, como verificado por Cruz (1974): planalto, escarpa e planícies litorâneas. Estes dois últimos compartimentos apresentam maior relavância na ocorrência dos movimentos de massa, entre eles, os escorregamentos e os fluxos de detritos, na Serra do Mar, pois caracterizam a zona de iniciação e de eposição destes processos.

A precipitação média anual em Caraguatatuba é de $1784 \mathrm{~mm}$ (SANTOS e GALVANI, 2012). De forma geral, o Litoral Norte de São Paulo possui alta variabilidade de precipitação, principalmente entre os meses de fevereiro e março, conforma Tavares et al. (2004). Segundo os autores, o município de Caraguatatuba apresenta uma menor variabilidade de precipitação, em relação aos demais municípios do Litoral Norte, devido a sua posição geográfica, mais recuada, que ameniza o efeito na relação da circulação atmósferica com a orografia, resultando nas sombras de chuva (TAVARES et al., 2004). 
NERY, T.D. O uso de parâmetros morfométricos como potencial ...

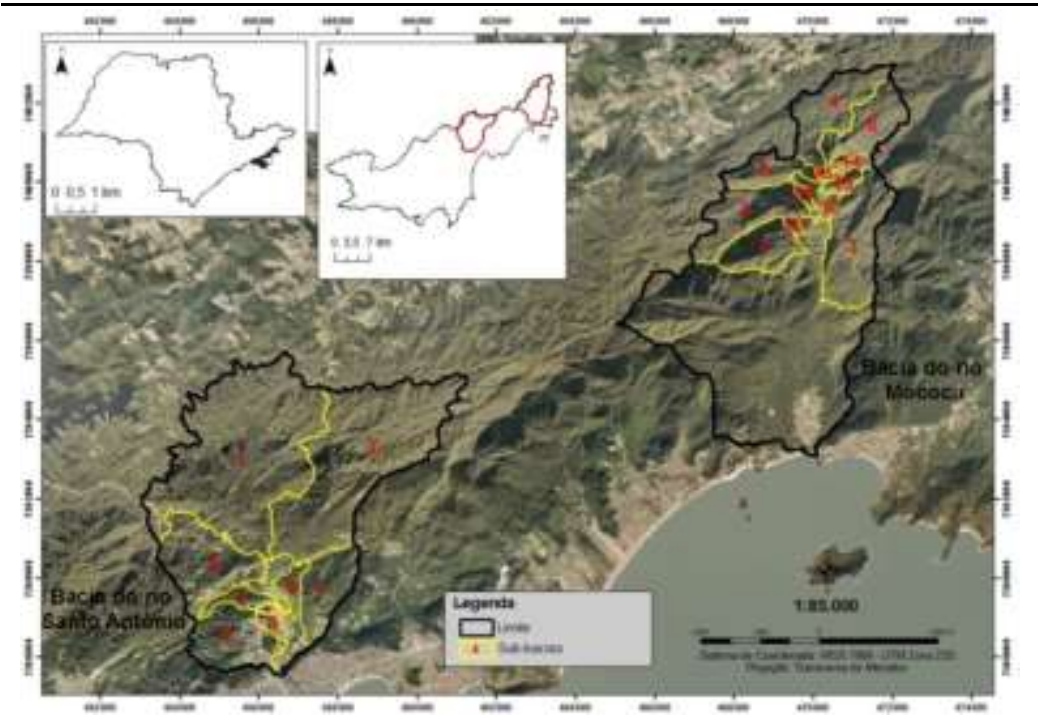

Figura 1: Localização das bacias dos rios Santo Antônio e Mococa, em Caraguatatuba (SP).

\section{Materiais e métodos}

Para o estudo foram selecionadas as bacias hidrográficas dos rios Santo Antônio e Mococa, com aproximadamente $40 \mathrm{~km}^{2}$ de área, localizadas na parte central e norte do município (Figura 1), devido ao comportamento diferenciado na ocorrência dos fluxos de detritos para o ano de 1967.

A partir da escolha destas bacias selecionou-se respectivamente, 8 (oito) sub-bacias do Santo Antônio e 12 (doze) sub-bacias do rio Mococa para a análise qualitativa e quantitativa quanto ao potencial de geração de corridas.

\section{Parâmetros morfométricos}

Os parâmetros morfométricos foram obtidos por meio do Modelo Digital de Terreno disponibilizado pela Empresa Paulista 
NERY, T.D. O uso de parâmetros morfométricos como potencial ...

de Planejamento Metropolitano (EMPALSA; Contrato de Licença de Uso - CLU N ${ }^{\circ}$ 049/14), em escala de $25 \mathrm{~m}^{2}$ e resolução de $1 \mathrm{~m}$.

Por meio do sistema TerraHidro aplicou-se uma rotina para a extração da rede de drenagem e, posteriormente a aquisição das variáveis utilizadas na análise dos fluxos de detritos: a área da bacia $\left(\mathrm{km}^{2}\right)$, que apresenta alta correlação com a vazão e o volume de sedimentos; a amplitude da bacia $(\mathrm{m})$, que indica a energia potencial necessária para o escoamento do material; o comprimento do canal $(\mathrm{km})$, que corresponde ao trecho em que o fluxo de detritos pode se desenvolver; a declividade média do canal $\left({ }^{\circ}\right)$, podendo indicar que quanto maior a declividade, maior será o fluxo da corrente; o índice de circularidade (adimensional), que corresponde ao tempo de concentração do escoamento dentro da bacia; e a densidade de drenagem $\left(\mathrm{km} / \mathrm{km}^{2}\right)$, este podendo contribuir na vazão e no potencial de inundação. Estas variáveis foram pautadas nos trabalhos de Augusto Filho (1993), Jackson (1987), IPT (2002), Coe et al. (2004), Wilford et al. (2004), Hajam et al. (2013) entre outros.

\section{Modelos empíricos e Parametrização de modelos}

A partir da aquisição de dados de entrada foram selecionados dois modelos empíricos de vazão de pico e volume total de sedimentos.

Para avaliar a vazão de pico dos fluxos de detritos o modelo escolhido para a análise foi o proposto por Araya Moya (1994) e modificado por Massad et al. (1997). A modificação no modelo corresponde na substituição da variável de precipitação em 24 horas $\left(I_{24}\right)$ pela intensidade de precipitação horária $\left(I_{1}\right)$, pois segundo Massad et al. (1997) a intensidade de precipitação antecedente ao evento é mais significativa na ocorrência dos fluxos de detritos na Serra do Mar, em São Paulo (Equação 1).

$$
q_{T}=1,4 \cdot \frac{1}{(1-c)} \cdot 0,85 \cdot A \cdot I_{1} \cdot \frac{H^{0,19}}{L_{0,58}} \text { Eq. } 1
$$


NERY, T.D. O uso de parâmetros morfométricos como potencial ...

Onde $c$ é a concentração de sedimentos, por unidade do volume $\left(\mathrm{m}^{3}\right), A$ é a área da bacia $\left(\mathrm{km}^{2}\right), I_{1}$ é a intensidade de precipitação horária que antecede o evento, $H$ é a diferença altimétrica (m) e $L$ é o comprimento do canal $(\mathrm{km})$.

Para estimar o volume total de sedimentos das corridas de detritos, adotou-se o modelo de Takahashi (1991). Este modelo foi construído por meio de observações a respeito dos fluxos de detritos em diferentes regiões do mundo, e é representado pela Equação 2:

$$
V_{\mathrm{t}}=500 \cdot q_{T} \text { Eq. } 2
$$

Onde, $V_{T}$, é o volume total de sedimentos, em $\mathrm{m}^{3} ; q_{T}$ é a vazão de pico do processo $\left(\mathrm{m}^{3} / \mathrm{s}\right)$.

Para avaliar o potencial de fluxo de detritos foram empregados diferentes valores de concentração de sedimentos e de intensidade de precipitação para a composição dos cenários. Os valores de concentração de sedimentos (c) foram obtidos na literatura por meio de observações de campo e em laboratório e varia entre $10 \%$ a $90 \%$ (COSTA, 1984; DAVIES et al., 1992; WHIPPLE e DUNNE, 1992; RICKNMANN e ZIMMERMANN, 1993; COUSSOT e MEUNIER, 1996; JISHAN e TIANCHI, 2001; GODT e COE, 2007; UEHARA et al., 2008; entre outros). Com o intuito de compreender o seu comportamento, no caso, o volume dos fluxos de detritos foram aplicados dois valores de concentração de sedimentos, de $30 \%$ e $50 \%$, como propostos por Gramani et al. (2005) e Kanji et al. (2008). Segundo Gramani et al. (2005), a concentração de 30\% representara os sedimentos grossos e que seriam responsáveis pela destruição das infraestruturas localizadas nas áreas onde ocorrem a passagem dos fluxos de detritos. Em contrapartida, o valor de $50 \%$, proposto por Kanji et al. (2008) corresponde à toda fração sedimentar do fenômeno, não distiguindo o diâmetro destes sedimentos.

A variável climática foi obtida por meio da retroanálise feita por IPT (2002) em função da análise da série histórica de precipitação da Baixada Santista e Litoral Norte de São Paulo 
NERY, T.D. O uso de parâmetros morfométricos como potencial ...

associada à ocorrência de eventos de fluxos de detritos. Neste estudo, verificou-se que as ocorrências destes processos na Serra do Mar, em São Paulo, estavam associadas a intensidades de precipitação de $60 \mathrm{~mm} . \mathrm{h}-1$ e $100 \mathrm{~mm} . \mathrm{h}-1$, correspondendo a tempos de retorno de 10 e 100 anos (IPT, 2002). Este tipo de análise também foi observado por Staley (2013) em estudos realizados na Califórnia, EUA.

O Quadro 1 apresenta as condições iniciais para a análise quantitativa para a obtenção dos valores estimados de vazão e volume total de sedimentos.

Quadro 1: Informações para a análise qualitativa e quantitativa das corridas de detritos.

\begin{tabular}{|c|c|c|}
\hline Parâmetros & Forma de aquisição & Unidade \\
\hline Área & SIG & $\mathrm{km}^{3}$ \\
\hline Amplitude & SIG & $\mathrm{m}$ \\
\hline Comprimento do canal & SIG & $\mathrm{km}$ \\
\hline Ângulo da encosta & SIG & ${ }^{\circ}$ \\
\hline Densidade de drenagem & Excel & $\mathrm{km} \cdot \mathrm{km}-2$ \\
\hline Índice de circularidade & Excel & adimensional \\
\hline \multirow{2}{*}{$\begin{array}{c}\text { Concentração de } \\
\text { sedimentos* }\end{array}$} & Gramani et al. (2005); & \multirow{2}{*}{$\%$} \\
\hline & Massad et al. (1997), & \\
\hline Precipitação (Cenário) & IPT (2002). & mm.h-1 \\
\hline
\end{tabular}

Ressalta-se, que o presente artigo embasou-se no evento de 1967, em Caraguatatuba, em São Paulo e, portanto, somente foram avaliadas as sub-bacias que foram identificados a ocorrência de escorregamentos e fluxo de detritos. Neste contexto, 8 de 9 subbacias do rio Santo Antônio e 12 de 36 sub-bacias do rio Mococa foram selecionadas para a análise qualitativa e quantitativa. 
NERY, T.D. O uso de parâmetros morfométricos como potencial ...

\section{Resultados e discussões}

\section{Análise Qualitativa - Parâmetros e Índices morfométricos}

Os resultados dos parâmetros morfométricos das 20 subbacias selecionadas podem ser observados nas Tabelas 1 e 2 . Os resultados indicam, para as sub-bacias selecionadas, os parâmetros e índices morfométricos que apresentam ou não condições, de contribuir na deflagração dos fluxos de detritos nas bacias do Santo Antônio e Mococa, tomando como cenário, o evento de 1967 em Caraguatatuba (SPS).

Tabela 1: Parâmetros morfométricos das sub-bacias selecionadas do rio Santo Antônio.

\begin{tabular}{ccccccc}
\hline $\mathrm{Sb}$ & Á & Amp & CRP & IC & DD & DMC \\
\hline 1 & 13,8 & 810 & 8,2 & 0,29 & 3,6 & 5,05 \\
2 & 10,9 & 790 & 8,2 & 0,34 & 4,2 & 6,04 \\
3 & 0,69 & 380 & 2,7 & 0,22 & 4,8 & 5,65 \\
4 & 0,96 & 310 & 1,5 & 0,39 & 4,5 & 8,77 \\
5 & 3,9 & 750 & 3,7 & 0,48 & 3,8 & 10,36 \\
7 & 2,3 & 340 & 2,9 & 0,55 & 4,3 & 6,36 \\
8 & 0,3 & 10 & 1,3 & 0,32 & 6,1 & 0,43 \\
9 & 2,8 & 570 & 2,6 & 0,53 & 3,6 & 8,08 \\
\hline
\end{tabular}

SB: Sub-bacias; A: Área $\left(\mathrm{km}^{2}\right)$; Amp: Amplitude (m); CRP: Comprimento do rio principal $(\mathrm{km})$; IC: Índice de circularidade (adm); DD: densidade de drenagem $(\mathrm{km} . \mathrm{km}-2)$; DMC: Declividade média do canal principal $\left(^{\circ}\right)$.

A área destas sub-bacias variou entre 0,3 e $13,8 \mathrm{~km}^{2}$, sendo os fluxos de detritos ocorridos nas sub-bacias 1,2 e 5 da bacia do rio Santo Antônio e nas sub-bacias 3 e 5 do rio Mococa. $\mathrm{Na}$ literatura, a ocorrência desses fenômenos está condicionada a bacias inferiores a $10 \mathrm{~km}^{2}$ (Mizuyama, 1982) e acima destes valores a sua ocorrência pode estar associada a outros processos, por exemplo, os escorregamentos (JAKOB, 1996). Neste sentido, a 
NERY, T.D. O uso de parâmetros morfométricos como potencial ...

compreensão destes processos é mais significativa, pois a relação entre a produção e a vazão de sedimentos, em escala de sub-bacias, ocorre de forma igualitária, em detrimento aos escoamentos das grandes bacias, principalmente os sedimentos mais grosseiros (MIZUYAMA, 1982; JACKSON, 1987). Ressalta-se, que em todas estas sub-bacias foram observadas cicatrizes de escorregamentos, porém não foi observado o desenvolvimento dos fluxos de detritos.

Tabela 2: Parâmetros morfométricos das sub-bacias selecionadas do rio Mococa.

\begin{tabular}{ccccccc}
\hline $\mathrm{Sb}$ & $\mathrm{A}$ & Amp & CRP & IC & DD & DMC \\
\hline 3 & 4,1 & 860 & 4,2 & 0,47 & 4,07 & 9,17 \\
4 & 2,9 & 620 & 4,5 & 0,34 & 3,8 & 6,80 \\
5 & 2,8 & 730 & 3,8 & 0,38 & 3,7 & 9,96 \\
6 & 2,2 & 600 & 3,4 & 0,40 & 3,7 & 8,51 \\
7 & 1,9 & 770 & 2,4 & 0,60 & 4,3 & 11,78 \\
9 & 1,3 & 560 & 2,7 & 0,36 & 3,2 & 10,68 \\
13 & 0,41 & 570 & 1,1 & 0,55 & 3,5 & 16,21 \\
14 & 0.33 & 510 & 1,2 & 0,41 & 3,8 & 20,47 \\
17 & 0,23 & 370 & 0,80 & 0,46 & 5,2 & 15,33 \\
21 & 0,17 & 600 & 0,73 & 0,38 & 5,09 & 12,28 \\
22 & 0,13 & 340 & 0,42 & 0,57 & 3,4 & 12,03 \\
33 & 0,12 & 430 & 0,55 & 0,45 & 5,2 & 20,86 \\
\hline $\mathrm{SB}$ & $\mathrm{Sub}-\mathrm{b}$ & & $\mathrm{A}: \mathrm{A}$ &
\end{tabular}

SB: Sub-bacias; A: Área $\left(\mathrm{km}^{2}\right)$; Amp: Amplitude (m); CRP: Comprimento do rio principal $(\mathrm{km})$; IC: Índice de circularidade (adm); DD: densidade de drenagem (km.km-2); DMC: Declividade média do canal principal $\left({ }^{\circ}\right)$.

Em relação à amplitude, estudos apontam que quanto maior a amplitude, maior será a energia potencial para o escoamento do material dos fluxos de detritos (IPT, 2002). Jackson (1987) aponta 
NERY, T.D. O uso de parâmetros morfométricos como potencial ...

que bacias favoráveis ao desenvolvimento destes processos são aquelas com valores superiores a $500 \mathrm{~m}$ : sub-bacias 9, 5, 2 e 1 (Santo Antônio) e as sub-bacias 14, 9, 13, 6, 21, 4, 5, 7 e 3 (Mococa). Considerando a declividade média do canal, as subbacias 5 (Santo Antônio) e 14, 13, 21, 7 e 9 (Mococa) apresentam maior potencial para a geração dos fluxos de detritos, pois este parâmetro influencia na capacidade de erosão e na (re) mobilização do material depositado ao longo da rede de drenagem (AUGUSTO FILHO, 1993). Contudo, outras sub-bacias, com amplitudes inferiores a $500 \mathrm{~m}$ mas com alto índice de declividade média do canal, também podem apresentar condições para a geração destes processos, sendo os casos das sub-bacias 33, 17 e 22 do rio Mococa.

Em contrapartida, quanto maior a declividade média do canal menor a probabilidade de deposição no leito em relação à extensão do canal, pois, segundo IPT (2002), quanto maior for o comprimento do canal, maior será o escoamento superficial e maior será a probabilidade de transporte e acúmulo de sedimentos em seu leito. Como observado na Tabela 2, os maiores valores de declividade foram associados à sub-bacias com comprimento de canal inferior a $1,2 \mathrm{~km}$, indicando que nestes canais pode haver uma menor taxa de deposição e um maior escoamento dos sedimentos para os canais subsequentes.

Sub-bacias com índices de circularidade próximo a 1 apresentam baixo tempo de concentração, indicando uma maior probabilidade de cheia do canal e podendo contribuir com a ocorrência das corridas de detritos, sem a necessidade da deflagração dos escorregamentos. Áreas nestas condições foram associadas às sub-bacias 7 e 9 (rio Santo Antônio) e 13, 22 e 7 (rio Mococa), porém para o evento de 1967 não foi observado a ocorrência dos fluxos de detritos nestas sub-bacias. Em contrapartida, estes processos foram observados nas sub-bacias 1, 2 e 5 (Santo Antônio) e 3 e 5 (Mococa), todas com índices entre 0,3 e 0,48 , porém associadas com índices de densidade de drenagem entre 3 e 4 . 
NERY, T.D. O uso de parâmetros morfométricos como potencial ...

Segundo Gartner et al. (2008) um alto valor de densidade de drenagem pode aumentar a magnitude do fluxo de detritos. Sobre este índice, Hajam et al. (2013) afirmaram que este parâmetro pode determinar o tempo de deslocamento do fluxo e, consequentemente o controle do escoamento superficial da água e dos sedimentos. De fato, isso pode explicar a ocorrência destes processos nestas subbacias, que somado à intensidade de precipitação, contribuiria para a elevação destes rios, permitindo escoar todos os sedimentos, provenientes das encostas e, principalmente do próprio canal.

\section{Análise Quantitativa - Potencial de geração de sedimentos}

Os resultados obtidos pelos modelos empíricos considerando os cenários de intensidade de precipitação $(60 \mathrm{~mm} . \mathrm{h}-1$ e $100 \mathrm{~mm} . \mathrm{h}$ 1) e de concentração de sedimentos (c) (30\% e 50\%) resultaram em uma diferença de $60 \%$ e $71 \%$, respectivamente (Figura 2 ).

Figura 2: Gráfico de vazão (V.P.) e volume de sedimentos (V.T.) em função da concentração de sedimentos.

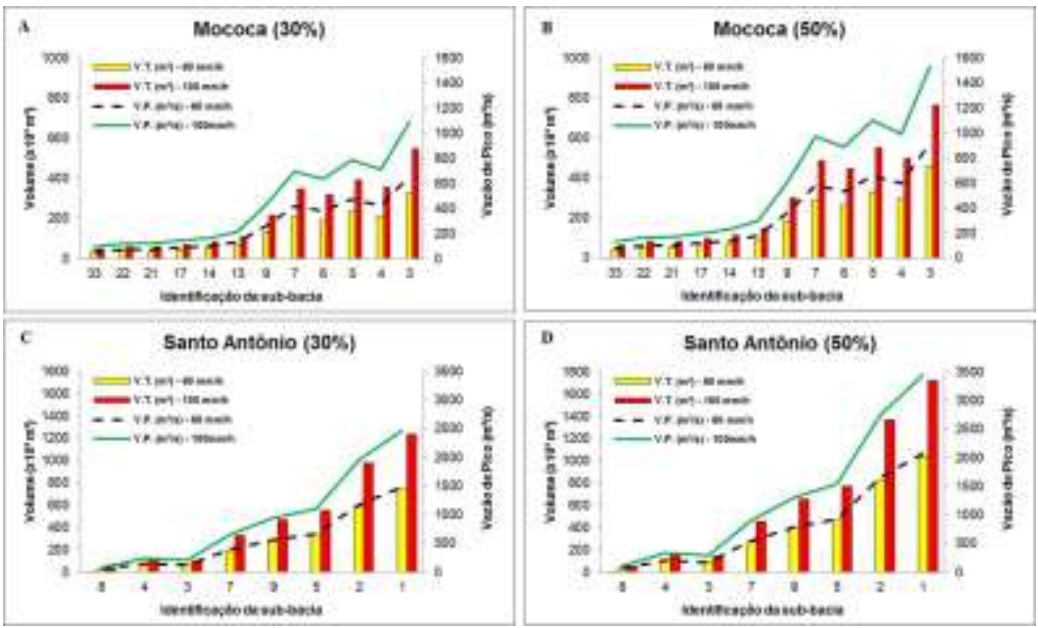


NERY, T.D. O uso de parâmetros morfométricos como potencial ...

Sub-bacias com áreas inferiores a $1 \mathrm{~km}^{2}$ em relação ao cenário de 60 mm.h-1 e com concentração de sedimentos de $50 \%$ podem gerar volumes de sedimentos de até $100.550 \mathrm{~m}^{3}$. Considerando a mesma concentração de sedimentos, porém com precipitação de $100 \mathrm{~mm}$.h-1, estas mesmas áreas gerariam volumes de sedimentos de até $167.583 \mathrm{~m}^{3}$. Estes volumes decrescem com a alteração da concentração de sedimentos (30\%), passando a volumes de sedimentos máximos para o cenário de $60 \mathrm{~mm} . \mathrm{h}-1 \mathrm{e}$ 100 mm.h-1 de $71.821 \mathrm{~m}^{3}$ e $119.702 \mathrm{~m}^{3}$, respectivamente.

As sub-bacias entre 2 e $3 \mathrm{~km}^{2}$ e concentração de sedimentos de $50 \%$, tiveram os volumes de sedimentos variando entre 200.000 $\mathrm{m}^{3}$ e $400.000 \mathrm{~m}^{3}$ para o cenário de $60 \mathrm{~mm} . \mathrm{h}-1$ e entre $440.000 \mathrm{~m}^{3} \mathrm{e}$ $652.000 \mathrm{~m}^{3}$ para o cenário de $100 \mathrm{~mm} . \mathrm{h}-1$. Em relação a concentração de sedimentos de $30 \%$ estes intervalos variaram entre $190.000 \mathrm{~m}^{3}$ e $280.000 \mathrm{~m}^{3}$ (cenário de $60 \mathrm{~mm} . \mathrm{h}-1$ ) e entre 317.000 $\mathrm{m}^{3}$ e $466.000 \mathrm{~m}^{3}$ (cenário de $100 \mathrm{~mm} . \mathrm{h}-1$ ).

As sub-bacias com áreas superiores a $10 \mathrm{~km}^{2}$ foram somente identificadas na bacia do rio Santo Antônio. O volume de sedimentos gerados por estas sub-bacias para a concentração de sedimentos de $50 \%$ variou entre $820.803 \mathrm{~m}^{3}$ e $1.033 .689 \mathrm{~m}^{3}$ (cenário de $60 \mathrm{~mm} . \mathrm{h}-1$ ) e $1.368 .005 \mathrm{~m}^{3}$ e $1.722 .815 \mathrm{~m}^{3}$ (cenário de 100 mm.h-1). Estes valores, como apontado anteriormente, representaram uma diferença de $71,4 \%$ em relação à concentração de sedimentos de $30 \%$.

Esta diferença na concentração de sedimentos, 30\% e 50\%, pode indicar o comportamento dos fluxos de detritos, contribuindo, por exemplo, no seu raio de alcance e no seu poder de impacto. Como verificado por Gramani et al. (2005) uma concentração de sedimentos de 30\% seria responsável por maiores danos às infraestruturas, por exemplo, os oleodutos e estações de captação de água localizados na Serra do Mar. Acredita-se que a trajetória destes materiais grosseiros seja reduzida ao longo do canal, associada também à diminuição da declividade média do canal ou até mesmo pela presençade rupturas contribuindo na acumulação destes e, escoando, assim, os sedimentos menos grosseiros. No 
NERY, T.D. O uso de parâmetros morfométricos como potencial ...

entanto, estes mesmos materiais podem contribuir em eventos futuros aumentando, assim, o poder de impacto destes processos. Salienta-se que este comportamento é inerente às particularidades de cada sub-bacia aqui analisada, ou seja, a disponibilidade de material proveniente dos escorregamentos e/ou disponíveis ao longo da rede de drenagem, deve ser considerada na análise.

Portanto, acredita-se que a adoção de $50 \%$ de concentração de sedimentos seja responsável por danos maiores, visto que, incorpora sedimentos de diâmetros variados e que são capazes de deslocarem-se até regiões mais planas. Exemplos desta dinâmica foram observados por Cruz (1974), Kanji et al. (2008) e Gramani (2013), os quais relataram a inundação de bairros e destruição de casas e pontes, bem como, a interrupção do Pólo Industrial e o corte no abastecimento de água a jusante das bacias na Serra do Mar, em Caraguatatuba e em Cubatão.

\section{Conclusões}

Os métodos qualitativos e quantitativos aplicados em escala de sub-bacias em relação aos fluxos de detritos permitiram identificar as áreas com maior capacidade de geração de volumes de sedimentos.

O método qualitativo apontou que sub-bacias com áreas inferiores a $3 \mathrm{~km}^{2}$, amplitudes superiores a $500 \mathrm{~m}$ e índice de circularidade superior a 0,5 podem apresentar maiores condições na geração das corridas de detritos. Para o evento de 1967, o índice de circularidade não apresentou nenhuma relação com a deflagração destes processos, visto que, não foi identificado a presença destes processos nas sub-bacias com os maiores valores para este índice. No entanto, ressalta-se que estes parâmetros são apenas um indicativo, pois outros fatores também podem influenciar na ocorrência destes processos, inclusive nas demais sub-bacias que não apresentaram estas características.

$\mathrm{O}$ método quantitativo (modelo empírico) identificou que a intensidade de precipitação e a concentração de sedimentos 
NERY, T.D. O uso de parâmetros morfométricos como potencial ...

influenciaram de forma significativa o volume de sedimentos gerados. Em relação à intensidade de precipitação houve um aumento de $60 \%$ no volume de sedimentos entre os cenários propostos. Quando analisados em função da concentração de sedimentos verificou-se que o volume de sedimentos aumentou $71 \%$. Ressalta-se, que a concentração de sedimentos observados na literatura diz respeito aos depósitos, ou seja, aqueles localizados ao longo do canal e aos leques aluvionares localizados nas áreas mais planas da bacia e, para uma melhor avaliação destes processos, bem como, dos modelos empíricos, observações a respeito desta variável devem levar em consideração os depósitos ao longo de toda trajetória.

Os resultados aqui encontrados podem subsidiar estudos de planejamento minimizando possíveis danos e/ou perdas de vidas, bem como, de infraestruturaslocalizadas dentro ou próximas das áreas onde as corridas de detritos se desenvolvem. Além disso, dados obtidos em campo podem auxiliar na calibração dos modelos empíricos, permitindo uma análise mais crítica dos resultados.

\section{Agradecimentos}

O autor agradece as contribuições de Marcelo Gramani, Milton Kanji, Claudinei T. Silveira pelas dicussões ao longo da construção desta pesquisa.

\section{Referências bibliográficas}

ARAYA MOYA, V. Genesis de aluviones en la costa de zonas deserticas. In: I Simposio Panamericano de Deslizamientos, 1994, Guayaquil, Equador. Anais, Guayaquil. p. 186-200.

AUGUSTO FILHO, O. O estudo das corridas de massa em regiões serranas tropicais: um exemplo de aplicação no município de Ubatuba, SP. In: Congresso Brasileiro de Geologia de Engenharia, 
NERY, T.D. O uso de parâmetros morfométricos como potencial ...

7., 1993, Poços de Caldas. Anais, Poços de Calda: ABGE, 1993, vol. 2 , p. 63-72.

AVELAR, A. S.; LACERDA, W. A.; COELHO NETO, A. L. Mecanismos de iniciação de fluxos detríticos no maciço da Tijuca, Rio de Janeiro (RJ): o caso da encosta do Soberbo. Revista Brasileira de Geomorfologia, 7, p. 73-83, 2006.

BRASIL. Lei $n^{\circ} 12.608$, de 10 de abril de 2012. Institui a Política Nacional de Proteção e Defesa Civil - PNPDEC; dispõe sobre o Sistema Nacional de Proteção e Defesa Civil-SINPDEC e o Conselho Nacional de Proteção e Defesa Civil - CONPDEC; autoriza a criação de sistema de informações e monitoramento de desastres; altera as Leis $\mathrm{n}^{\mathrm{os}} 12.340$, de $1^{\underline{\mathrm{o}}}$ de dezembro de 2010 , 10.257, de 10 de julho de 2001, 6.766, de 19 de dezembro de 1979, 8.239 , de 4 de outubro de 1991, e 9.394, de 20 de dezembro de 1996; e dá outras providências. Disponível em: http://www.planalto.gov.br/ccivil_03/_Ato20112014/2012/Lei/L12608.htm. Acessado em: agosto de 2015.

CANNON, S.H., GARTNER, J.E. (2005). Wildfire-related debris flows from a hazards perspective In: Hungr, O., Jakob, M. (Eds.), Debris-flow Hazards and Related Phenomenon. Praxis PublishingLtd, Chichester, UK, p. 1-17.

COROMINAS, J. The angle of reach as a mobility index for small and large landslides. Canadian Geotechnical Journal, v. 33, p. 260-271, 1996.

COSTA, J. E. Physical geomorphology of debris flows. In Costa, J. E., and Fleisher, J. P., (eds.), Developments and applications of geomorphology, New York: Springer-Verlag. p. 268-317, 1984 
NERY, T.D. O uso de parâmetros morfométricos como potencial ...

COUSSOT, P.; MEUNIER, M. Recognition, classification and mechanical description of debris flows. Earth-Science Reviews, v. 40, p. 209-227, 1996.

CRUZ, O. A Serra do Mar e o Litoral na área de Caraguatatuba - SP. 1972. p. 181. Tese (Doutorado em Geografia) - Faculdade de Filosofia, Letras e Ciências Humanas, Universidade de São Paulo, São Paulo, 1974.

DAVIES, T. R.; PHILLIPS, C. J.; PEARCE, A. J.; ZHANG, X. B. Debris flow behavior - an integrated overview. Erosion, Debris Flows and Environment in Moutain Regions. IAHS Publ., n209, p. 217-225, 1992.

DOWLING, C. A.; SANTI, P. M. Debris flows and their toll on human life: a global analysis of debris-flow fatalities from 1950 to 2011. Natural Hazards, v. 71, p. 203-227, 2014.

GARTNER, J. E.; CANNON, S. H.; SANTI, P. M.; DEWOLFE, V. G. Empirical models to predict the volumes of debris flows generated by recently burned in the western U.S. Geomorphology, v. 96, p. 339-354, 2008.

GODT, J. W.; COE, J. A. Alpine debris flows triggered by a 28 July 1999 thunderstorn in the central Front Range, Colorado. Geomorphology, v. 84, p. 80-97, 2007.

GRAMANI, M. F.; OLIVITO, J. P. R.; AUGUSTO FILHO, O.; MAGALHÃES, F.S.; Análise da potencialidade de geração de corridas de massa nos trechos serranos do duto OSBAT. In: Congresso Brasileiro de Geologia de Engenharia e Ambiental, 11, Florianópolis - SC. Anais... Florianópolis: CBGE, 2005 (CDROM). p. 1-15. 
NERY, T.D. O uso de parâmetros morfométricos como potencial ...

GRAMANI, M. F. A corrida de detritos (debris flow) no ribeirão Cágado, Serra do Mar, município de Cubatão, SP. In: VI Conferência Brasileira de Encostas, 2013. Anais...Angra dos Reis, Rio de Janeiro: COBRAE, 2013. p. 88-95.

GRAMANI, M. A corrida de massa no córrego Guarda-Mão, município de Itaoca (SP): Impacto e observações de campo. In: Congresso Brasileiro de Geologia de Engenharia e Ambiental, $15^{\circ}$, Bento Gonçalves - RS. Anais...Bento Gonçalves: CBGE, 2015 (CD-ROM), p. 1-10.

HAJAM, R. A.; HAMID, A.; BHAT, S. Application of morphometric analysis for geohydrological studies using geospatial technology - A case study of Vishav drainage basin. Hydrology Current Research, vol 4, n³, p. 1-12, 2013.

INSTITUTO DE PESQUISA TECNOLÓGICAS DO ESTADO DE SÃO PAULO. Reconhecimento, caracterização e monitoramento de locais potencialmente sujeitos a instabilizações na Serra do Mar na área de influência dos diversos Sistemas de Captação e Abastecimento de Água e de Tratamento de Esgoto. São Paulo, 2002. Relatório 59.123.

JACKSON, L. E. Debris flows hazard in the Canadian Rocky Mountains. Geological Survey of Canada, Paper 86-11, p. 20, 1987

JAKOB, M. Morphometric and geotechnical controls of debris flow: frequency and magnitude in southern British Columbia. (1996). P. 242. Tese (Doutorado em Filosofia) - Departamento de Geografia, Universidade de Columbia Britânica.

JISHAN, W.; TIANCHI, L. Behaviour and characteristics of debris flows. in: Tianchi, L., Chalise, S. R., and Upreti, B. N. 
NERY, T.D. O uso de parâmetros morfométricos como potencial ...

(eds), Landslide Hazard Mitigation, ICIMOD, Kathmandu, p. 203-2014, 2001.

KANJI, M. A.; CRUZ, P. T.; MASSAD, F. Debris flow affecting the Cubatão Oil Refinery, Brazil. Landslides, v. 5, p. 71-82, 2008

MARCHI, L.; D'AGOSTINO, V. Estimation of debris-flow magnitude in the Eastern Italian Alps. Earth Surface Processes and Landforms, v. 29, p. 207-220, 2004.

MASSAD, F., CRUZ, P. T., KANJI, M. A., ARAUJO FILHO, H. A. Comparison between estimated and measured debris flows discharges and volume of sediments. In: 2nd Panamerican Symposium on Landslide e $2^{\circ}$ Congresso Brasileiro de Estabilidade de Encosta - COBRAE, 1997 ABMS/ABGE, Rio de Janeiro, 1997: p. 213-222.

MIZUYAMA, T. Analysis of sediment yield and transport data for erosion control works. Recent Developments in the Explanation and Prediction of Erosion and Sediment Yield. Proceedings Exeter Symposium, July: 82, IAHS Publ., n 137, p. 177-182, 1982.

PELLERIN, J.; DUARTE, G. M.; SCHEIBE, L. F.; MENDONÇA, M.; BUSS, M. D.; MONTEIRO, M. A. Timbé do Sul - Jacinto Machado: Avaliação preliminar da extensão da catástrofe de 2324/12/1995. Geosul, v. 12 (23), p. 71-86, 1997.

RICkENMANN, D.; ZIMMERMANN, M. The 1987 debris flows in Switzerland: documentation and analysis. Geomorphology, v.8, p. 175-189, 1993.

RICKENMANN, D. Empirical relationships for debris flows. Natural Hazards, v. 19, p. 47-77, 1999. 
NERY, T.D. O uso de parâmetros morfométricos como potencial ...

ROSS, J. L. S.; MOROZ, I. C. Mapa Geomorfológico do Estado de São Paulo. Laboratório de Geomorfologia. São Paulo: Departamento de Geografia - FFLCH - USP/Laboratório de Cartografia Geotécnica - Geologia Aplicada - IPT/FAPESP (Fundação do Amparo à Pesquisa do Estado de São Paulo), 1997. (Mapas e Relatórios).

SANTOS, D. D.; GALVANI, E. Caracterização sazonal das precipitações no município de Caraguatatuba - SP, entre os anos de 1943 a 2004. Revista GEONORTE, Edição Especial 2, vol. 1, n5, p. 1196-1203, 2012.

STALEY, D. M. Staley, D.M. Emergency assessment of post-fire debris-flow hazards for the 2013 Rim Fire, Stanislaus National Forest and Yosemite National Park, California: U.S. Geological Survey Open-File Report 2013-1260, 11 p., 3 plates. Disponível em: http://pubs.usgs.gov/of/2013/1260/ Acesso em: 17/02/2016

TAKAHASHI, T. Estimation of potential debris flows and their hazardous zones: soft countermeasures for a disaster. Journal of Natural Disaster Science, v. 3, nº 1, p. 57-89, 1981.

TAKAHASHI, T. Debris flow. Rotterdam; Brookfield Published for the International Association for Hydraulic Research by A.A. Balkema: 1991, p. 164

TAVARES, R.; SANT'ANNA NETO, J. L.; TOMMASSELLI, J. T. G.; PRESSINOTTI, M. M. N.; SANTORO, J. Análise da variablidade temporal e espacial das chuvas associada aos movimentos de massa no Litoral Norte Paulista. In: Simpósio Brasileiro de Desastres Naturais, 1., 2004, Florianópolis. Anais... Florianópolis: GEND/UFSC, 2004. p. 680-696. (CD ROM) 
NERY, T.D. O uso de parâmetros morfométricos como potencial ...

WHIPPLE, K. X; DUNNE, T. The influence of debris-flow rheology on fan morphology, Owens Valley, California. Geological Society of America Bulletin, v. 104, p. 887-900, 1992.

WILFORD, D. J.; SAKALS, M. E.; INNES, J. L.; SIDLE, R. C.; BERGERUD, W. A. Recognition of debris flow, debris flood and flood hazard through watershed morphometrics. Landslides, vol. 1, p. 61-66, 2004. 